\title{
Native Aortic Valve Endocarditis caused by Neisseria elongata in a Previously Healthy Patient
}

\author{
Sara Haddad $^{1}$, Ralph Tayyar ${ }^{1}$, Phillip Gary ${ }^{1}$, Jerome Santoro ${ }^{2}$, Brett Gilbert ${ }^{2}$ \\ ${ }^{1}$ Internal Medicine Department and ${ }^{2}$ Infectious Diseases Division, Lankenau Medical Center, Wynnewood, PA, United States.
}

\section{Corresponding Author: \\ Dr Ralph Tayyar \\ Email: rtayyar@stanford.edu}

This is an Open Access article distributed under the terms of the Creative Commons Attribution License (creativecommons.org/ licenses/by/3.0).

Received Accepted Published

December 10, 2020

February 4, 2021

March 25, 2021

\begin{abstract}
Background: Neisseria elongata can behave as an opportunistic infection and can cause an array of diseases, including destructive endocarditis. Around $60 \%$ of cases of $N$. elongata infective endocarditis require surgical intervention. We report a case of $N$. elongata native aortic valve endocarditis in a previously healthy patient with no known risk factors who required valve replacement. Case Report: A 63-year-old female with no risk factors presented with fevers, headache and anorexia and was found to have Neisseria elongata endocarditis. Transesophageal echocardiography showed a $1.5 \times 1.5$ $\mathrm{cm}$ vegetation on aortic valve. Patient did well after aortic valve replacement surgery and a 6-week course of ceftriaxone. Conclusion: N. elongata endocarditis should be thought off in endocarditis cases, even with no apparent risk factors and cardiothoracic surgical evaluation is imperative due to destructive nature of this disease.
\end{abstract}

Keywords: Aortic Valve, Endocarditis, Fever, Heart Valve Prosthesis, Neisseria elongata.

\section{Introduction}

Neisseria elongata is a gram-negative rod-shaped bacterium that lives in the normal flora of the oropharynx [1]. The genus elongata is divided into three sub-species differentiated by biochemical characteristics: $N$. elongata, N. glycolytica, and $N$. nitroreducens [2]. The below report mentions $N$. elongata as a genus and not as a sub-species. This organism has been associated with a variety of infectious diseases including bacteremia and endocarditis [3]. Known major risk factors for $N$. elongata endocarditis are dental manipulations, previous history of endocarditis or valve damage, and rheumatic heart disease [4]. N. elongata has been identified as cause of endocarditis on any of the heart valves. Reported complications include valve root abscess, pseudoaneurysm, and heart failure in a surprisingly high proportion of cases [5]. We report a case of $N$. elongata native aortic valve endocarditis in a previously healthy patient with no known risk factors who required valve replacement.

\section{Case Report}

A 63-year old female with no pertinent past medical history presented to the emergency department for evaluation of intermittent fever of three weeks duration. She had associated headache, lethargy, vomiting, and decreased oral intake. She denied chest pain, palpitations, shortness of breath, joint pain, or rash. She had no recent dental manipulations, travel or sick contacts. She was not sexually active. On arrival, the patient was noted to be slow to respond, oriented to place and self only. She was febrile with a temperature of $102.9^{\circ} \mathrm{F}$, heart rate of 109 beats/minute, and blood pressure of $69 / 48 \mathrm{mmHg}$. She had dry mucous membranes, dentition was intact, neck was supple, and no rash was noted. Heart exam revealed sinus tachycardia with a grade 2 systolic ejection murmur, lung and abdominal exams were unremarkable. No focal neurologic signs were found. She had no evidence of heart failure or stigmata suggestive of endocarditis. Initial laboratory testing was pertinent 
for leucocytosis and abnormal liver function tests [Table 1]. Influenza A and B antigen testing and urine analysis were negative as well as viral hepatitis panel and mononucleosis testing. Chest X ray and $\mathrm{CT}$ brain were normal. $\mathrm{CT}$ abdomen and pelvis with intravenous contrast revealed no definite source of infection. Two sets of blood cultures were obtained and she was admitted to the intensive care unit for close monitoring and management. She did not respond to fluid resuscitation and required initiation of pressor therapy. She was initially started on ceftriaxone, vancomycin, and intravenous methyl-prednisolone for suspicion of bacterial meningitis. Acyclovir was added to cover for possible herpes encephalitis. With supportive measures, the patient's mental status improved; she became conversant and cooperative, and was oriented to place, person, and time. Both sets of blood cultures were growing gram negative bacilli identified the following day as Neisseria elongata.

The patient was continued on intravenous ceftriaxone $2 \mathrm{~g}$ daily and all other antimicrobials were discontinued. Repeat blood cultures cleared on hospital day two. Initial transthoracic echocardiogram showed mild aortic valve regurgitation with a suspicious underlying vegetation. A transesophageal echocardiogram showed a large $1.5 \times 1.5 \mathrm{~cm}$ mass attached to the non-coronary cusp of the aortic valve in addition to aortic regurgitation [Fig.1]; no other cardiac abnormality was noted. She underwent aortic valve replacement using a $23 \mathrm{~mm}$ bioprosthetic valve. Pathology of the native aortic valve showed acute inflammatory infiltrates with tissue cultures growing Neisseria elongata. She was continued on ceftriaxone $2 \mathrm{~g}$ daily for a total course of 6 weeks as an outpatient. To date, two months after the surgery, patient remains afebrile, doing well with no evidence of infection recurrence.

\section{Discussion}

The genus Neisseria includes $N$. gonorrhoeae and $N$. meningitidis, the two most well-known human
Table 1: Laboratory Workup.

\begin{tabular}{|l|l|l|l|l|}
\hline & Reference range & $\begin{array}{l}\text { Admission } \\
\text { day }\end{array}$ & Day 2 & $\begin{array}{l}\text { Discharge } \\
\text { day }\end{array}$ \\
\hline Hemoglobin & $11.8-15.7 \mathrm{~g} / \mathrm{dL}$ & $\mathbf{1 0 . 2}$ & $\mathbf{1 0 . 1}$ & $\mathbf{1 0 . 9}$ \\
\hline White blood cells & $3.80-10.50 \mathrm{~K} / \mu \mathrm{L}$ & $\mathbf{1 8 . 2}$ & 9.3 & 7.2 \\
\hline Platelet count & $150-369 \mathrm{~K} / \mu \mathrm{L}$ & $\mathbf{1 4 2}$ & 196 & 299 \\
\hline BUN & $8-20 \mathrm{mg} / \mathrm{dL}$ & 15 & 15 & 11 \\
\hline Creatinine & $0.6-1.1 \mathrm{mg} / \mathrm{dL}$ & 0.7 & 0.7 & 0.6 \\
\hline Sodium & $136-144 \mathrm{mEq} / \mathrm{L}$ & $\mathbf{1 3 0}$ & 139 & 139 \\
\hline Potassium & $3.6-5.1 \mathrm{mEq} / \mathrm{L}$ & $\mathbf{3 . 2}$ & 3.7 & 4.5 \\
\hline $\begin{array}{l}\text { Aspartate } \\
\text { aminotransferase }\end{array}$ & $15-41 \mathrm{IU} / \mathrm{L}$ & $\mathbf{1 1 9}$ & $\mathbf{6 6}$ & 28 \\
\hline $\begin{array}{l}\text { Alanine } \\
\text { aminotransferase }\end{array}$ & $11-54 \mathrm{IU} / \mathrm{L}$ & $\mathbf{1 8 4}$ & $\mathbf{1 3 1}$ & 38 \\
\hline $\begin{array}{l}\text { Alkaline } \\
\text { phosphatase }\end{array}$ & $35-126 \mathrm{IU} / \mathrm{L}$ & $\mathbf{2 8 5}$ & $\mathbf{2 6 4}$ & 102 \\
\hline
\end{tabular}

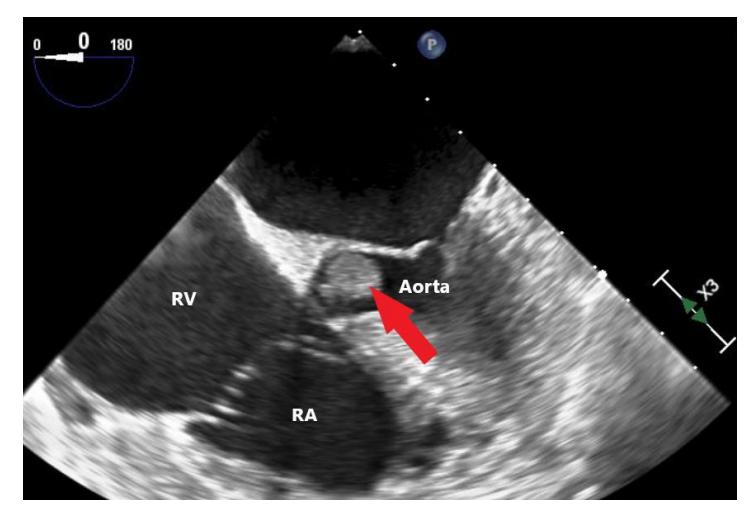

Fig.1: Transesophageal echocardiogram showing the $1.5 \times 1.5 \mathrm{~cm}$ vegetation (red arrow) on the aortic valve. $R A$ : right atrium; $R V$ : right ventricle.

pathogens. However, emerging case reports are suggesting that the more commensal species of Neisseria, including $N$. elongata, can also behave as opportunistic pathogens and cause an array of diseases [6]. N. elongata has particularly been associated with invasive endocarditis with notable sequelae such as brain embolization and abscess formation, myocardial abscess [7], and mycotic aneurysms [8]. These complicated courses of $N$. elongata endocarditis may in part be attributed to the fact that this organism is often not considered to be a destructive pathogen due to its commensal nature. 
Pre-existing heart disease, valvular or congenital, is considered a risk factor for $N$. elongata endocarditis [2]. Some cases have been reported in patients with no apparent cardiac disease but with other co-morbidities or recent dental infections or procedures [4]. To our knowledge, this case of $N$. elongata endocarditis constitutes an atypical presentation of the entity as it occurred in a previously healthy patient with no apparent risk factors.

A recent review of the literature concerning $N$. elongata endocarditis shows that $60 \%$ of patients with this infection were noted to require surgical intervention, mostly because of the development of complications such as congestive heart failure, systemic emboli, and abscess formation [1]. In our patient, the main indication for surgical intervention was the large size of the vegetation. One would normally regard $N$. elongata as a commensal organism likely to present in a subacute fashion. However, although our patient was having intermittent symptoms for 3 weeks, she presented with leukocytosis and no other evidence of a prolonged infection suggesting that this organism can present in an acute manner. This presentation suggests that $N$. elongata can present acutely as a destructive pathogen.

\section{Conclusion}

Although $N$. elongata endocarditis rarely occurs without predisposing factors, this organism should be thought of as a cause of endocarditis even in previously healthy patients. It can lead to destructive infective endocarditis that often requires surgical intervention as well as a prolonged antibiotic therapy [5]. Noting the destructive nature of $N$. elongata endocarditis as demonstrated by our review of the literature and our experience with this patient, surgical intervention might need to be pursued early and more aggressively when dealing with this pathogen.

Contributors: SH, RT: manuscript writing, and patient management; PG: manuscript editing, references and patient management; JS, BG: critical inputs into the manuscript. RT will act as a study guarantor. All authors approved the final version of this manuscript and are responsible for all aspects of the study.

Funding: None; Competing interests: None stated.

\section{References}

1. Youssef D, Marroush TS, Levine MT, Sharma M. Endocarditis due to Neisseria elongata: A case report and review of the literature. Germs. 2019;9(4):188-192.

2. Rossella P, Patrizia C, Oreste TF, Renato T, Emanuele G, Vincenzo A, et al. Native mitral valve endocarditis caused by Neisseria elongate subsp. nitroreducens in a patient with Marfan syndrome: First case in Italy and review of the literature. Case Rep Infect Dis. 2016;4956205.

3. Grant PE, Brenner DJ, Steigerwalt AG, Hollis DG, Weaver RE. Neisseria elongata subsp. nitroreducens subsp. nov. Formerly CDC Group M-6, a Gram-negative bacterium associated with endocarditis. J Clin Microbiol. 1990;28(12):2591-2596.

4. Wong JD, Janda JM. Association of an important Neisseria species, Neisseria elongate subsp. nitroreducens, with bacteremia, endocarditis, and osteomyelitis. J Clin Microbiol. 1992;30(3):719-720.

5. Haddow LJ, Mulgrew C, Ansari A, Miell J, Jackson G, Malnick H, et al. Neisseria elongata endocarditis: case report and literature review. Clin Microbiol Infect. 2003;9(5):426-430.

6. Humbert MV, Christodoulides M. Atypical, yet not infrequent, infections with Neisseria species. Pathogens. 2019;9(1):10.

7. Hsiao JF, Lee MH, Chia JH, Ho WJ, Chu JJ, Chu PJ. Neisseria elongata endocarditis complicated by brain embolism and abscess. J Med Microbiol. 2008;57 (Pt 3):376-381.

8. Nawaz T, Hardy DJ, Bonnez W. Neisseria elongata subsp. elongata, a cause of human endocarditis complicated by pseudoaneurysm. J Clin Microbiol. 1996;34(3):756-758. 